InnOvaciOnes de NegOciOs 7(1): 065 -088, 2010

(c) 2010 UANL, Impreso en México

\title{
Creatividad organizacional como un factor clave en el proceso de gestión de la innovación para el desarrollo de nuevos productos, sector de estudio las PyMEs (Organizational creativity as a key element in the process of innovation management for new product development, SME sector study)
}

\author{
Oscar López, Mónica Blanco \& Sergio Guerra \\ UANL, San Nicolás de los Garza, N.L., México, lopezoscarenrique@yahoo.com.mx
}

Key words: CANIETI, Innovation, New Product Development (NPD), Organizational Creativity, SMEs.

\begin{abstract}
The purpose of this study is to analyze organizational creativity as a key element in the process of product innovation. Analyzing some manage models for organizational creativity presented by various authors who have studied this phenomenon. Also, this element of Organizational Creativity stems from a doctoral study in which this variable is considered as the contribution to a new management model for the innovation process in developing new products for the SME sector. The analysis of results and conclusions are derived from a study of 34 samples to the industry of information technology in Nuevo León, México.
\end{abstract}

Palabras clave. CANIETI, Creatividad Organizacional, Desarrollo de Nuevos Productos (DNP), Innovación, PyMEs.

Resumen. El propósito del estudio es analizar a la creatividad organizacional como un elemento clave en el proceso de innovación de producto. Se analizan los modelos existentes para gestionar a la creatividad organizacional, presentando las diferentes propuestas por distintos autores que han estudiado este fenómeno. Así mismo, este elemento de Creatividad Organizacional se deriva de un estudio doctoral en el que esta variable es considerada como el aporte a un nuevo modelo de gestión para el proceso de innovación en el desarrollo de nuevos productos para el sector de las PyMEs. El análisis de resultados y las conclusiones se presentan a partir de un estudio de 34 muestras a la industria de las tecnologías de la información en Nuevo León, México. 


\section{Introducción}

En la primera parte del documento se indaga sobre el tema de la innovación, su importancia y la propuesta de un nuevo modelo de gestión de innovación para las PyMEs. La segunda parte abarca el análisis de los diferentes modelos de gestión de la creatividad a través del tiempo, su importancia e impacto en el ámbito empresarial. Este último elemento o variable, es el aporte al modelo de gestión de innovación propuesto en la primera parte del artículo. Se termina con análisis de resultados y conclusiones sobre esta variable a partir de una muestra aplicada a la industria de las tecnologías de información, obtenida de la CANIETI (Cámara Nacional de la Industria Electrónica de Telecomunicaciones y Tecnologías de la Información) en la zona de N.L., México.

\section{Metodología.}

La creatividad de los individuos es el punto de partida para la innovación, es la semilla para crear innovación (Amabile y otros 1996). La relación entre creatividad e innovación lleva a gestionar adecuadamente a las empresas que desean ser innovadoras. Aunque la creatividad organizacional es un elemento indispensable en las organizaciones, no siempre es fácil obtenerla ya que algunos estilos de liderazgo pueden inhibir a que se dé este elemento y por ende, la innovación se ve afectada.

Muchos autores señalan que la creatividad es la materia prima de la innovación visto desde la perspectiva de los negocios y que ésta se debe, en cierta manera, de gestionar para obtener resultados positivos en la resolución de problemas. Así mismo, la innovación es, para muchos países, parte básica de su crecimiento económico y orgánico. Se han generado muchos estudios alrededor de estos dos grandes temas tanto en el ámbito laboral como en el académico.

Por ejemplo, el reporte anual de la encuesta global sobre la innovación organizacional realizada por el Boston Consulting Group a 1070 ejecutivos de empresas representando a 63 países nos revela que (BCG, 2006):

- El $72 \%$ de los ejecutivos consideraron a la innovación dentro de las 3 principales estrategias del negocio.

\section{O. López, M. Blanco \& S. Guerra}


- El $42 \%$ consideraron a la innovación como su principal estrategia.

- El 52\% están satisfechos con el retorno de inversión de la innovación.

- El 90\% consideran relevante a la innovación para el desarrollo del crecimiento organizacional y para el éxito de la industria.

El presente estudio se deriva de la inquietud de abordar el tema de Innovación analizándolo en el contexto de las PyMEs, pero sobre todo el análisis de la Creatividad Organizacional, ya que es un elemento clave dentro del modelo de Innovación que más adelante se abordará.

En el tema de los modelos de gestión de la innovación, se puede mencionar que han existido muchos autores (desde la segunda guerra mundial hasta la fecha) que concentran, en forma de modelo de gestión, sus conocimientos acerca de la concepción de la innovación en el producto. En este artículo se han concentrado estos modelos de gestión en cinco grandes generaciones de los diferentes autores y especialistas en el tema. Cada generación de modelos está descrita por ciertos factores o elementos que de forma secuencial y/o inter-relacional conforman cada uno de los diagramas 0 modelos propuestos.

Se estudiarán los elementos que conforman cada uno de los modelos, y se seleccionarán algunos para integrarlos en uno nuevo modelo propuesto enfocado a las PyMEs, como la propuesta de valor de este artículo. La razón de dirigirse a este sector es por la importancia que tiene en la economía de cualquier país en el mundo, y más para los que están en desarrollo, como el caso de México.

Ya una vez seleccionadas las variables para construir el nuevo modelo propuesto, se le adiciona el constructo de la creatividad organizacional, ya que este elemento es considerado vital para que la innovación se dé en el desarrollo de nuevos productos. Cabe señalar que ningún modelo de gestión estudiado hasta el momento plasma este constructo como parte vital del proceso de innovación de producto. El aporte para la investigación en curso es este último constructo de Creatividad Organizacional como proceso operacional.

A su vez, en este documento, se exploran los diferentes procesos para generar el pensamiento creativo que se han sugerido en la literatura en los últimos 80 años. De igual manera, se estudian los modelos de gestión de

\section{Creatividad Organizacional \& PyMEs}


la creatividad propuestos por los diferentes autores desde los 1930's hasta los 1990's y se concentran en una matriz comparativa de variables, como soporte a la importancia del constructo de Creatividad Organizacional.

Planteamiento del problema de investigación.

¿Es la Creatividad Organizacional un elemento clave para el proceso de gestión de la Innovación en el desarrollo de nuevos productos para el sector PyMEs?

Objetivo general y específicos de investigación.

Analizar e Integrar a la Creatividad Organizacional como un elemento clave a la propuesta de modelo de gestión de la Innovación que permite mejorar el proceso de desarrollo de nuevos productos en el sector PyMEs.

1.- Integrar la Creatividad Organizacional a un modelo propuesto para PyMEs.

2.- Elaborar y aplicar la encuesta a una muestra seleccionada a fin de obtener la importancia relativa individual de la creatividad organizacional.

3.- Analizar los resultados, establecer conclusiones y recomendaciones que permitan validar el impacto de la creatividad organizacional al modelo propuesto.

Hipótesis de la investigación.

La creatividad e innovación deben ser fruto de una actividad sistemática para obtener mayor éxito en la idea de valor. La innovación no es una actividad aislada, sino un proceso total que está formado por la interrelación de subprocesos para conformar un modelo de gestión. La innovación es el futuro del desarrollo económico del país, y por tanto como nación, México debe crear productos de mayor valor agregado.

En base a las investigaciones analizadas la hipótesis de investigación para este estudio se afirma que la creatividad organizacional es uno de los elementos clave que conforman un modelo de gestión de la Innovación en las PyMEs que permitan desarrollar productos innovadores, así como lo son también la Orientación al Mercado, Investigación y Desarrollo, Diseño del Producto, Eficiencia Operacional y Eficiencia Comercial.

\section{O. López, M. Blanco \& S. Guerra}




\section{Marco teórico de la investigación.}

Antes de empezar a profundizar en el tema de PyMEs en México y abordar el tema de Innovación, se hace referencia que el presente estudio se deriva de la inquietud en aportar a la pequeña y mediana empresa una herramienta más que le permita aumentar su competitividad dentro de un mercado cada día más agresivo y complejo, como en el que está inmerso.

Como se dijo anteriormente, se han concentrado cinco generaciones de modelos de gestión de la innovación, por lo que a medida que avanza la forma de concebir y desarrollarse el conocimiento externo e interno a la organización y los efectos que produce, se han suscitado cambios sustanciales en los modelos conceptuales de innovación.

Del análisis de las investigaciones realizadas por diferentes autores, se tomará para el estudio las que el autor Roy Rothwell ha concentrado en estos años. Este investigador destaca que los modelos más destacados son los Modelos Lineales, Ios Modelos por Etapas, Ios Modelos Interactivos, los Modelos Integradores y el Modelo en Red. Se muestra a continuación cada uno de ellos para entrar a conocer las variables que lo conforman y de esta perspectiva, la propuesta de valor del estudio en curso iniciará a conformarse.

1- Modelos Lineales: Impulso de la Tecnología y Tirón de la Demanda.

Ambos se caracterizan por su concepción lineal del proceso de innovación. La innovación tecnológica está descrita como un proceso de conversión, en el que unos inputs se convierten en productos a lo largo de una serie de pasos (Forrest, 1991 en Velasco, 2005). Su principal característica es su linealidad, que supone un escalonamiento progresivo, secuencial y ordenado desde el descubrimiento científico (fuente de la innovación), hasta la investigación aplicada, el desarrollo tecnológico, la fabricación y el lanzamiento al mercado de la novedad.

En la figura 1, se muestra ambos modelos que aparecieron a mediados de los 1940's hasta principios de los 1960's. Esta época fue un período en el que la lucha de las grandes corporaciones por una mayor participación en el mercado se vio acompañada de un creciente énfasis estratégico en la mercadotecnia. Como consecuencia de todo ello, la

\section{Creatividad Organizacional \& PyMEs}


percepción del proceso de innovación comenzó a verse alterada, produciéndose una mayor intensificación de los factores de la demanda (Rothwell, 1994 en Velasco, 2005).

Figura 1: Modelo de Empuje de la Tecnología y Tirón de la Demanda

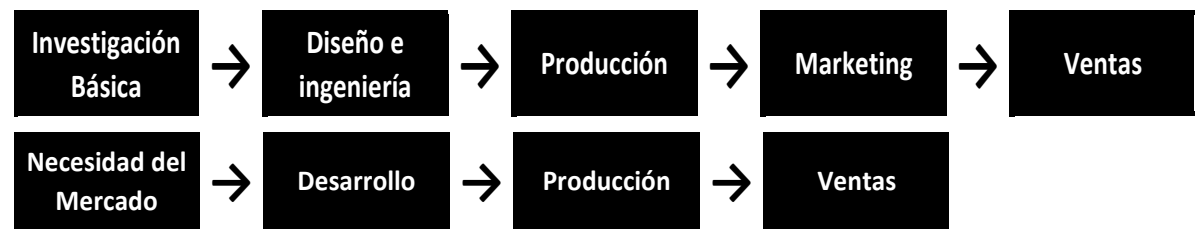

Fuente: Rothwell, R. (1994, p. 8-9) en Velasco, 2005.

\section{2- Modelos por Etapas.}

Estos modelos, al igual que los anteriores y que surgieron durante los 1960's, consideran la innovación como una actividad secuencial de carácter lineal. Se contempla el proceso de innovación como una serie de etapas consecutivas, detallando y haciendo énfasis, en las actividades particulares que tienen lugar en cada una de las etapas, así como en los departamentos involucrados (Forrest, 1991 en Velasco, 2005). Describe asimismo el proceso de innovación en términos simples, pero añade una etapa de actividad más. La generación de una idea, por parte del departamento de I\&D.

En la figura 2, se definen las cinco etapas, y se establece que el proceso de retroalimentación tiene lugar entre los mismos departamentos. (Saren, 1984 en Velasco, 2005).

Figura 2. Modelo por etapas departamentales

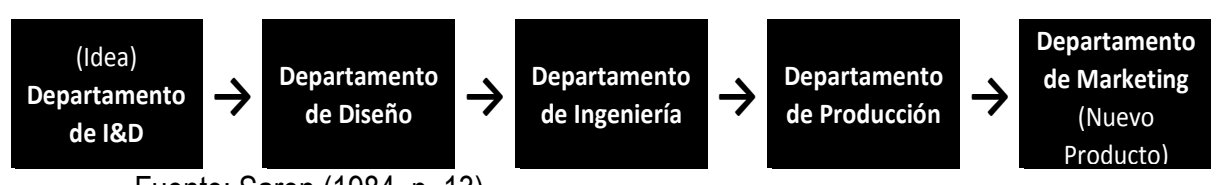

Fuente: Saren (1984, p. 13)

3- Modelos Interactivos o Mixtos.

Los Modelos Interactivos, se desarrollan durante los 1970's y son considerados por las empresas como una mejor práctica al proceso de

\section{O. López, M. Blanco \& S. Guerra}


innovación. Fue una época asociada a elevadas tasas de inflación y desempleo, unidas a una saturación de la demanda, por lo que las estrategias de las empresas estaban dirigidas a la racionalización y control de costos. La necesidad de entender la lógica del proceso de innovación y las bases de las innovaciones exitosas es imperiosa, para conseguir reducir la incidencia de fallos y el despilfarro de recursos (Rothwell, 1994 en Velasco, 2005).

En la figura 3, el modelo de Kline, en lugar de tener un único curso principal de actividad como el modelo lineal, tiene cinco caminos 0 trayectorias que son vías que conectan las tres áreas de relevancia en el proceso de innovación tecnológica: la investigación, el conocimiento y la cadena central del proceso de innovación tecnológica (Kline y Rosenberg, 1986 en Velasco, 2005). El camino central comienza con una idea que se materializa en un invento. El segundo trayecto consiste en una serie de retroalimentaciones donde el círculo pequeño da respuesta y que conecta cada fase de la cadena central con su fase previa (Kline, 1986 en Velasco, 2005).

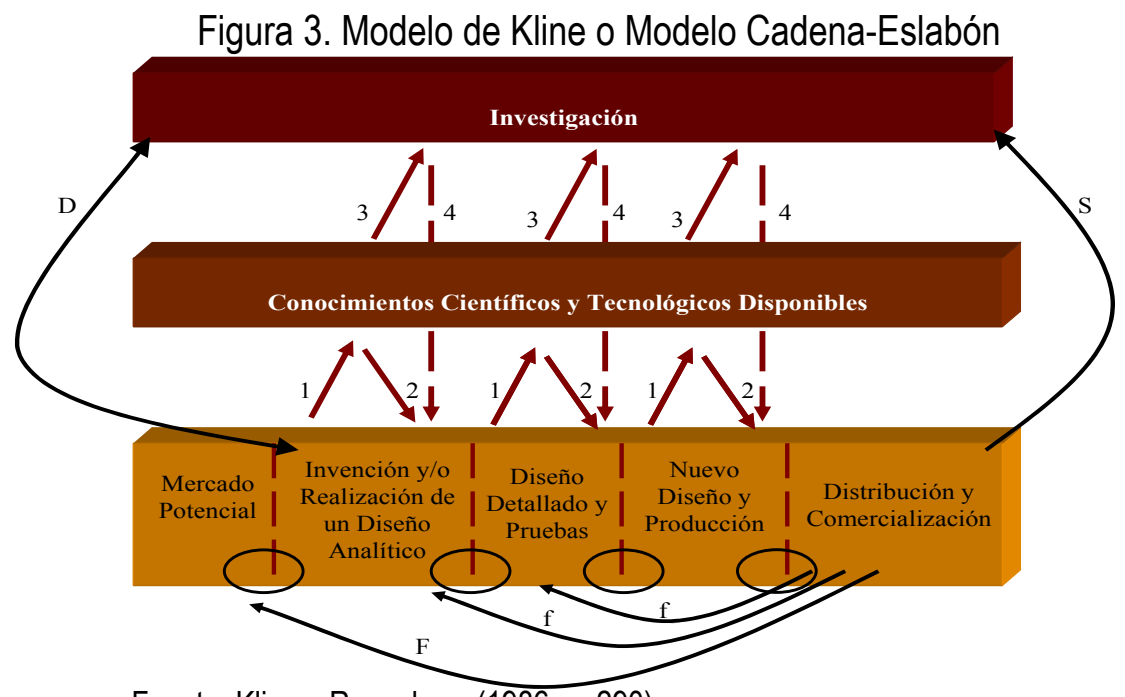

Fuente: Kline y Rosenberg (1986, p. 290)

4- Modelos Integradores.

Aparecen en los 1980 's, cuando las empresas siguen la tendencia a

Creatividad Organizacional \& PyMEs 
centrarse en la esencia del negocio y en las tecnologías básicas. La estrategia de las empresas es establecer todo tipo de alianzas estratégicas, además que el ciclo de vida de los productos es cada vez menor, hace que la velocidad de desarrollo se imponga como un factor clave para competir, empujando a las empresas a adoptar estrategias basadas en el tiempo de respuesta para lanzar productos al mercado (Hobday, 2005 en Velasco, 2005).

Se hace una analogía de estos modelos de innovación al llamado "enfoque rugby", que contrasta el punto de vista tradicional de carácter secuencial y representa la idea de un grupo que, como unidad, trata de desarrollar una distancia, pasando la bola hacia atrás y hacia delante (Takenuchi y Nonaka, 1986). Bajo este enfoque, el proceso de desarrollo de producto tiene lugar en un grupo multidisciplinario cuyos miembros trabajan juntos desde el comienzo hasta el final.

Figura 4. Modelo de fases de desarrollo de producto secuenciales

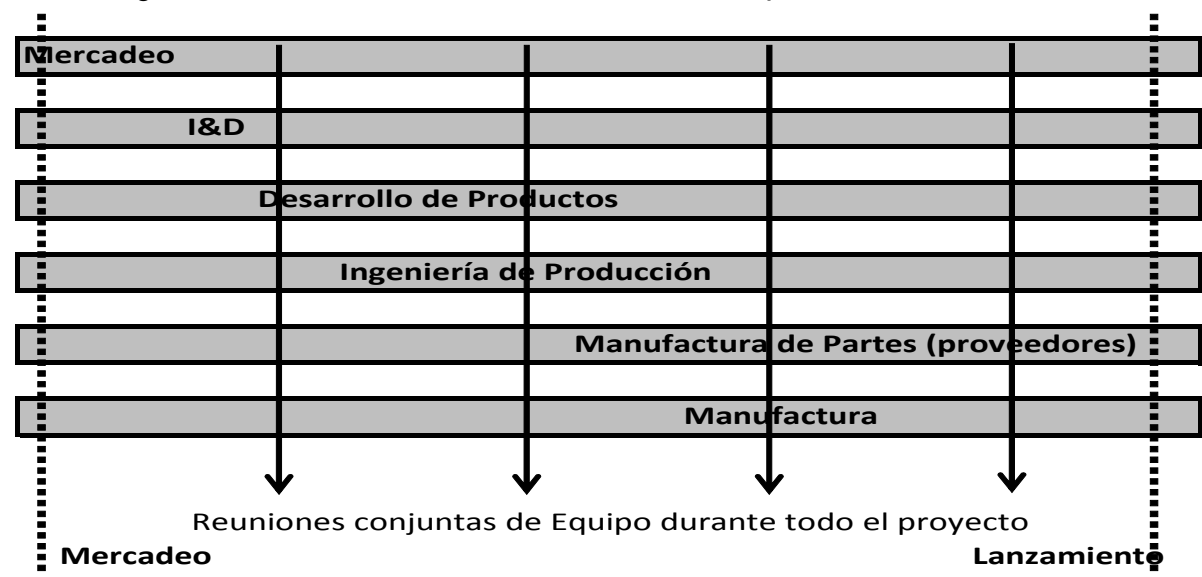

Fuente: Takeuchi, H. y Nonaka, I. (1986, p. 139)

5- Modelo en Red.

El modelo de integración de sistemas y establecimiento de redes es conocido como el modelo de quinta generación, a partir de mediados 1990's. El aprendizaje tiene lugar dentro y entre las empresas, sugiriendo que la innovación es generalmente un proceso distribuido en red.

\section{O. López, M. Blanco \& S. Guerra}


Las tendencias estratégicas observadas en la década de los ochenta continúan produciéndose en los noventa, pero con mayor intensidad: las compañías líderes siguen comprometidas con la acumulación tecnológica; las empresas continúan estableciendo redes estratégicas; la velocidad por llegar al mercado sigue siendo un factor de competitividad clave; persisten los esfuerzos por lograr una mejor integración entre las estrategias de producto y las de producción (diseño para la manufactura); las empresas muestran cada vez una mayor flexibilidad y adaptabilidad (organizacional, productiva y en productos); y las estrategias de producto enfatizan la calidad y el rendimiento. Se empieza a ver el desarrollo de Marcas más que de productos. (Rothwell, 1994 en Velasco, 2005).

En la figura 5, se visualiza que el conocimiento externo a la organización proviene de muchas fuentes y que el trabajo interno es coordinado todo el tiempo a los largo del proyecto. Este modelo se caracteriza por la utilización de sofisticadas herramientas electrónicas que permiten a las empresas incrementar la velocidad y la eficiencia en el desarrollo de nuevos productos, tanto internamente (actividades funcionales), como externamente (red de proveedores, clientes y colaboradores externos).

Figura 5: Modelo en RED

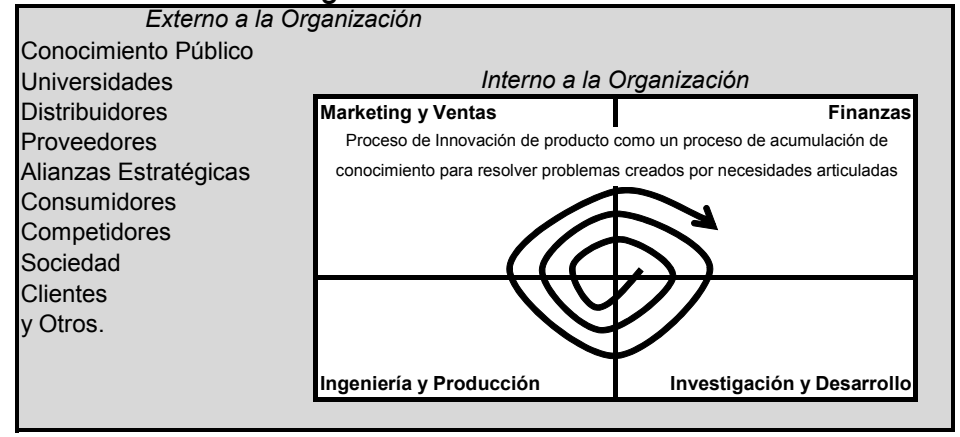

Fuente: Trott (1998), citado en Hobday (2005, p. 126)

En base a lo visto en estas cinco generaciones de modelos de gestión de la innovación se plantea un nuevo modelo, a partir de las variables que conforman éstos, pero enfocado a las PyMEs. Por lo que este modelo no es tan sofisticado, pero si lo suficientemente robusto para que apoye la creación de productos innovadores en las empresas de este sector.

\section{Creatividad Organizacional \& PyMEs}


A continuación se plasma en forma de Modelo los elementos clave para desarrollar nuevos productos innovadores en el sector PyMEs.

En la figura siguiente, se indica lo que se había dictado al inicio del estudio, a este nuevo modelo se está adicionando el constructo de creatividad organizacional ya que ningún modelo teórico lo ha propuesto. De cierta manera se puede pensar que la creatividad es una actividad cognoscitiva que se da por un hecho en cada unos de los procesos de la organización, pero la realidad es que el tema de creatividad es tan extenso que hay muchos autores y estudios que aseveran que éste es un proceso muy importante que se debe de gestionar y medir, y que tiene que verse desde una perspectiva de un proceso operacional como lo es la cadena de suministro o de cobranza para una compañía.

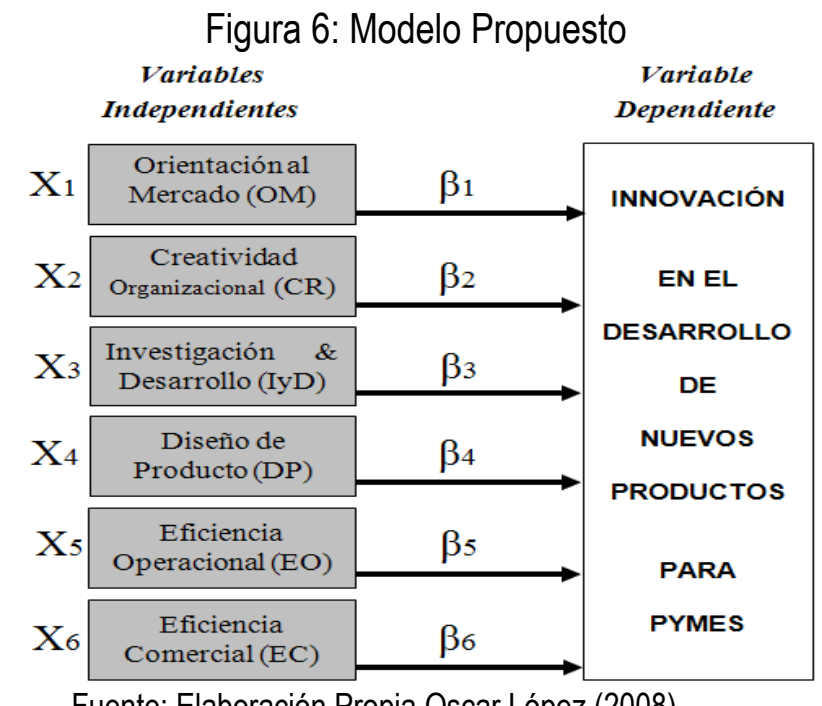

A continuación se presentan los conceptos y las definiciones de las variables del Modelo propuesto:

1. Orientación al Mercado (OM): cultura organizacional que de manera más eficiente y efectiva cree los comportamientos necesarios a fin de generar un valor superior para los compradores. Fuente: Narver y Slater (1990).

\section{O. López, M. Blanco \& S. Guerra}


2. Creatividad Organizacional (CR): es la producción de ideas diferentes y utilizables por un individuo o grupo pequeño de individuos trabajando juntos. Fuente: Amabile, Teresa (1988).

3. Investigación y Desarrollo (lyD): comprende todo el trabajo creativo llevado a cabo sobre una base sistemática en orden a incrementar el stock de conocimiento incluyendo las tres actividades fundamentales que lo conforman: investigación básica, investigación aplicada y desarrollo experimental. Fuente: Manual de Frascati, elaborado por la OCDE en su 6ta edición en 2002.

4. Diseño de Producto (DP): es el servicio profesional de crear y desarrollar conceptos y especificaciones que optimicen la función, valor o apariencia del producto y sistemas para el beneficio mutuo entre el usuario final y el manufacturador. Fuente: Industrial Design Society of America (IDSA); (www.idsa.org).

5. Eficiencia Operacional (EO) [Manufactura y Finanzas]: es un conjunto de operaciones que sirven para mejorar e incrementar la utilidad o el valor de los bienes y servicios económicos de una compañía. Fuente: Elaboración Propia.

6. Eficiencia Comercial (EC) [Distribución y Comercialización]: conjunto de actividades desarrolladas con el fin de facilitar la venta de una mercancía 0 un producto en una compañía. Fuente: Elaboración Propia.

Es tiempo de profundizar más en la variable de Creatividad Organizacional y justificar el porqué debe ser considerada en este nuevo modelo de gestión de innovación para las PyMEs.

En los 1950's fueron particularmente prolíficos para la investigación en el área de la creatividad, ya que es en 1950 cuando P.J. Guilford, para entonces presidente de la Asociación Americana de Psicología (APA), propone analizar más a fondo el tema al revelar el escaso porcentaje de artículos publicados referidos al mismo en relación con otros tópicos como la inteligencia. Señaló Guilford que de 121,000 artículos publicados en el Psychological Abstracts entre 1920 y 1950, sólo la cantidad de 186 se referían a creatividad (Guilford, 1971).

A partir de ese momento se incrementa el interés de muchos investigadores por el estudio de este constructo. El entorno, ambiente o clima

\section{Creatividad Organizacional \& PyMEs}


organizacional, es un factor decisivo en el desarrollo y expresión de la creatividad. De allí que muchos investigadores hayan orientado sus estudios hacia la influencia de estos aspectos en la creatividad organizacional.

A continuación se presenta una serie de teorías que enmarcan los modelos de gestión de la creatividad que pretenden explicar el proceso de creación y que serán la base fundamental para justificar este constructo dentro del modelo propuesto en esta investigación.

Uno de los primeros modelos del proceso de creación se atribuye a Graham Wallas en 1926, y propone que el producto del pensamiento creativo se genera a través de cuatro fases.

Modelo Wallas 1926 (proceso de cuatro pasos):

1. Preparación (definición del problema, la observación y estudio).

2. De incubación (por el que se la cuestión de lado por un tiempo).

3. Iluminación (el momento en que una nueva idea surge finalmente).

4. Verificación (control hacia fuera).

Torrance (1988) afirma que el modelo de Wallas es la base, en la actualidad, para la mayoría de los programas de formación de pensamiento creativo. La inclusión de incubación seguida de una iluminación repentina en este popular modelo puede explicar porqué el pensamiento creativo es considerado por mucha gente como un proceso mental subconsciente, que no puede ser dirigida.

El autor Rossman examinó el proceso creativo a través de cuestionarios completados por 710 inventores, tomando como base el modelo original de Wallas pasando de cuatro pasos a siete.

Modelo Rossman 1931 (modelo de siete pasos):

1. La observación de una necesidad o dificultad.

2. Análisis de la necesidad.

3. Un estudio (o encuesta) de toda la información disponible.

4. Una formulación de todas las soluciones objetivo.

5. Un análisis crítico de estas soluciones para sus ventajas y desventajas.

6. El nacimiento de las nuevas idea o de las invenciones.

7. La selección y experimentación para probar la solución más prometedora.

\section{O. López, M. Blanco \& S. Guerra}


En este modelo se envuelve el "nacimiento de la idea nueva" en el misterio, sus pasos previos y posteriores a este momento de iluminación son claramente analíticos.

Otro gran pensador creativo fue Alex Osborn, que en 1953, desarrolló el concepto de "lluvia de ideas", plasmó una teoría de equilibrio entre el análisis y la imaginación en su modelo de siete pasos para el pensamiento creativo.

Modelo Osborn 1953 (modelo para el pensamiento innovador):

1. Orientación (hacia arriba, el problema).

2. Preparación (recopilación de datos pertinentes).

3. Análisis (degradación de los materiales correspondientes).

4. Ideación (acumular alternativas a través de las ideas).

5. Incubación (parar, para invitar a la iluminación).

6. Síntesis (juntar las piezas).

7. Evaluación (juzgar las ideas resultantes).

Este modelo implica la idealización de un propósito y sobre éste nace el concepto de "apilar alternativas" a dicho objetivo, y desarrollarlas a través de las reglas del conocido método de la lluvia de ideas (brainstorming) como herramienta para hacerlo.

Ciertos estudios sobre la creatividad individual y organizacional dieron al siguiente autor ciertos elementos para formar su modelo de gestión.

Modelo Amabile 1983 (proceso de pensamiento creativo):

1. Presentación de la Tarea.

2. Preparación de la Idea.

3. Generación de la Idea.

4. Validación de la Idea.

5. Evaluación del Resultado.

El autor representa su modelo haciendo la analogía sobre el proceso de nacimiento de un recién nacido.

Modelo Barron 1988 (proceso de creación psíquica):

1. Concepción (en una mente preparada).

2. Gestación (tiempo, estrechamente coordinados).

3. Parto (el sufrimiento de nacer, la aparición a la luz).

4. La crianza del recién nacido (nuevo período de desarrollo). 
Dicho modelo apoya la opinión popular de la creatividad como un proceso de participación de pensamientos subconscientes que escapan al control del creador. En contraste con el papel destacado que algunos modelos dan a los procesos subconscientes, Perkins (1981) argumenta que los procesos de subconsciente mental están detrás de todo pensamiento $\mathrm{y}$, por tanto, no desempeñan ningún papel extraordinario en el pensamiento creativo.

Se puede destacar que sólo porque no puede describir nuestros procesos de pensamiento no significa que no estamos en control de ellos. Además, Perkins dice, sólo porque los eventos aleatorios desempeñan un papel en algunos actos de creación, esto no debe interpretarse en el sentido que los sucesos aleatorios son la fuente de todos los actos de creación. Weisberg's (1993) hace una revisión de la vida de los grandes creadores y de los llamados "momentos de la invención" y apoya los puntos de Perkins, demostrando que los años de trabajo consciente y preparación por parte del creador son la causa del acto creativo. Mientras que algunos modelos de la que parece que la creatividad es un proceso algo mágico, los modelos predominantes tienden más hacia la teoría de que las nuevas ideas surgen de un esfuerzo consciente para equilibrar el análisis y la imaginación.

La combinación sistemática de técnicas de creatividad dirigida y técnicas de análisis continúa siendo un tema fuerte en varios modelos más recientemente propuestos. Parnes (1992) y Isaksen y Trefflinger (1985) resumen seis pasos en la solución creativa de su popular solución de problemas (CPS) del modelo. (Decenas de miles de personas han aprendido el modelo de CPS y sus herramientas asociadas a través de los seminarios organizados por la Fundación de Educación Creativa en Buffalo, NY).

Modelo de CPS 1992 (solución creativa de problemas):

1. Comprobación del objetivo.

2. Determinación de los hechos.

3. La detección del problema.

4. Encontrar la idea.

5. La búsqueda de soluciones.

6. Búsqueda de aceptación.

Los pasos tres y cuatro (problema y encontrar la idea) exigen claramente el pensamiento creativo, mientras que los pasos restantes requieren conocimientos tradicionales y de pensamiento analítico.

\section{O. López, M. Blanco \& S. Guerra}


Los autores Koberg y Bagnall (1981) proponen un modelo equilibrado similar en su popular libro: El Viajero Universal.

Modelo de Koberg y Bagnall 1981 (EI Viajero Universal):

1. Aceptar la situación (como un desafío).

2. Analizar (para descubrir el "mundo del problema").

3. Define (las principales cuestiones y objetivos).

4. Idear (para generar las opciones).

5. Seleccione (a elegir entre las opciones).

6. Implementar (para dar forma física a la idea).

7. Evaluar (para examinar y planificar de nuevo).

Una vez más, se observa que la ideación, el enfoque tradicional de las herramientas de pensamiento creativo como la lluvia de ideas, se procedió y seguido por el pensamiento analítico y práctico deliberado. Koberg y Bagnall aceptan la situación como un desafío personal. Esto es consistente con la investigación sobre la vida de los grandes creadores que ilustra la importancia de centrar la atención y el cuidado profundamente. (Véase, por ejemplo, Weisberg 1993, Wallace y Gruber 1992, Gardner 1994, y Ghiselin 1952). Por último, el paso final de este modelo de apoyo a la noción de la innovación continúa.

El tema del equilibrio creativo y analítico se realiza en más de modelos propuestos para aplicaciones específicas. Bandrowski en 1985 considera a esto un proceso de planificación estratégica creativa. creativa):

Modelo Bandrowski 1985 (modelo de planeación estratégica

1. Planeación estándar.

2. Desarrollo de la visión o intuición.

3. Saltos creativos.

4. Conexiones estratégicas creativas.

5. Construcción del concepto.

6. Juicio crítico del concepto.

7. Planeación de la acción.

8. Planeación de la contingencia creativa.

9. Implementación flexible.

10. Monitoreo de los resultados

Se observa el papel positivo de la sentencia en este modelo y la 
necesidad de la aplicación específica en el desarrollo de habilidades creativas: visión, saltos creativos y planes de contingencia creativa.

Por último, es importante señalar que no todos los modelos colocan la generación de nuevos conceptos en la mente, si no que lo complementan con pensamiento analítico y ordenado. Por ejemplo, el "jamón" (paso de pensamiento creativo) del sándwich se coloca entre dos rebanadas de pan (pasos de pensamiento analítico). La creatividad de esa manera se considera que se debe de gestionar ordenadamente para alcanzar mejores resultados.

Robert Fritz 1991 (modelo para el proceso de la creación):

1. Concepción

2. Visión

3. La realidad actual

4. Tomar acciones

5. Ajustar, aprender, evaluar, ajustar

6. Construyendo el momento

7. Finalización del momento

8. Viviendo con tu creación

El autor identifica el inicio del proceso, como los actos creativos de la concepción y la visión. Es seguido por el análisis de la realidad actual, acción, evaluación, control público (impulso de construcción), y la terminación. Fritz, además, afirma que el proceso de creación es de naturaleza cíclica. "Vivir con tu creación" significa darse cuenta del propósito y análisis que conduce a la concepción creativa y visión próxima.

En la tabla 2, se muestra el resultado del análisis de las diferentes propuestas, a forma de resumen, y además de la relación de las variables de los modelos gestión de la creatividad. Se coloca una "X" para visualizar el proceso.

Independientemente del modelo específico en el cual se haya preferido, se está llamado a participar en este proceso mental durante un período prolongado de tiempo. La complejidad que implica este acto de equilibrio es probablemente la razón por la cual las ideas creativas son tan raras.

Para este estudio, se propone utilizar el modelo de Creatividad Organizacional expuesto por Amabile, para introducirlo dentro del nuevo modelo de gestión de la innovación para el desarrollo de nuevos productos

\section{O. López, M. Blanco \& S. Guerra}


en el sector de las PyMEs, ya que el autor ha dedicado a estudiar muchos años esta variable y tiene un gran número de artículos relacionados sobre el impacto positivo que tiene ésta en la organización cuando se gestiona y se mide.

Tabla 2. Matriz Comparativa de los Modelos de Gestión de Creatividad

\begin{tabular}{|c|c|c|c|c|c|c|c|c|c|}
\hline Grandes Rubros & \begin{tabular}{|l|} 
Modelo de \\
"Wallas 1926"
\end{tabular} & $\begin{array}{c}\text { Modelo de } \\
\text { "Rossman } \\
\text { 1931" } \\
\end{array}$ & $\begin{array}{l}\text { Modelo de } \\
\text { "Osborn 1953" } \\
\text { (brainstorming) }\end{array}$ & \begin{tabular}{|c|} 
Modelo de \\
"Kobergy \\
Bagnall 1981" \\
\end{tabular} & \begin{tabular}{|l|} 
Modelo de \\
"Amabile 1983" \\
\end{tabular} & $\begin{array}{c}\text { Modelo de } \\
\text { "Bandrowski } \\
\text { 1985" } \\
\end{array}$ & \begin{tabular}{|l|} 
Modelo de \\
"Barron 1988" \\
\end{tabular} & $\begin{array}{l}\text { Modelo de } \\
\text { "Fritz } 1991 " \\
\end{array}$ & $\begin{array}{l}\text { Modelo de "Parnes } \\
\text { 1992, Isaksen y } \\
\text { Trefflinger 1985" }\end{array}$ \\
\hline $\begin{array}{c}\text { Orientación / Comprobación } \\
\text { de le la dea }\end{array}$ & & & $\mathrm{X}$ & $x$ & $x$ & $x$ & & $x$ & $x$ \\
\hline $\begin{array}{l}\text { Preparacaín / Concepción de } \\
\text { la ldea }\end{array}$ & $x$ & $\mathrm{X}$ & $X$ & & $\mathbf{X}$ & $\mathrm{X}$ & $\mathrm{X}$ & $x$ & \\
\hline Análisis de Necesidad & & & $\mathrm{X}$ & $\mathrm{X}$ & & $x$ & & $x$ & $x$ \\
\hline $\begin{array}{l}\text { Incubación/ Gestación// } \\
\text { Estudio de Soluciones }\end{array}$ & $x$ & $x$ & $x$ & $x$ & $x$ & & $x$ & $x$ & $x$ \\
\hline lluminación / Parto & $\mathrm{x}$ & $\mathrm{x}$ & & $x$ & $x$ & $\mathrm{x}$ & $\mathrm{x}$ & & $x$ \\
\hline \begin{tabular}{|c|} 
Formulación / Idealización de \\
Soluciones \\
\end{tabular} & & $\mathrm{x}$ & $\mathrm{x}$ & $\mathrm{X}$ & $x$ & & & $\mathrm{x}$ & \\
\hline Construcción del Concepto & & & & & & $\mathrm{X}$ & & $\mathrm{X}$ & \\
\hline $\begin{array}{l}\text { Análisis Critico de las } \\
\text { Soluciones/ / Sintesis }\end{array}$ & & $\mathrm{X}$ & $\mathrm{X}$ & & $x$ & $\mathrm{X}$ & & & $\mathrm{X}$ \\
\hline \begin{tabular}{|c|} 
Planeacióne Implementar \\
Solución
\end{tabular} & & & & $x$ & & $\mathrm{X}$ & & $x$ & \\
\hline \begin{tabular}{|c|} 
Verfificación/Crianzal I \\
Aceptación de la Solución
\end{tabular} & $X$ & $x$ & $X$ & $X$ & & $x$ & $\mathrm{X}$ & $x$ & $\mathrm{X}$ \\
\hline
\end{tabular}

Fuente: Elaboración Propia.

De hecho Amabile, es una de los autores que expresa que la creatividad organizacional se debe de ver como un proceso operacional y que se debe de tratar como tal en las organizaciones.

En la figura 7, las flechas punteadas indican la influencia de los tres componentes sobre las cinco etapas y las flechas sólidas indican la secuencia en una sola dirección de los cinco pasos que componen el modelo de gestión de la creatividad, y lo que representa cada uno de ellos. Además de la interacción de los tres componentes (que se explican adelante) necesarios para la generación de la creatividad individual y/o de grupos pequeños. 
Figura 7: Modelo de Teresa Amabile

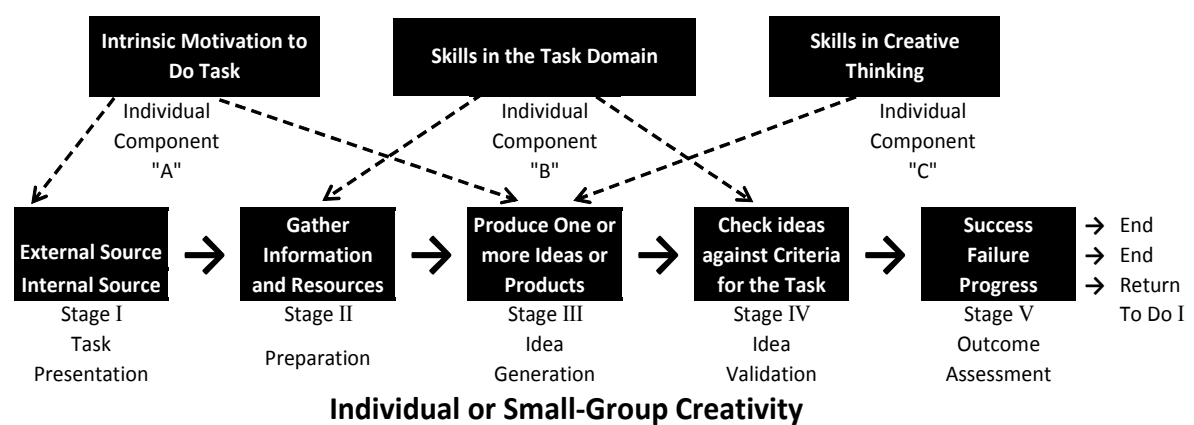

Fuente: Modelo de Creatividad (Amabile 1983).

Como lo define Amabile (1988), la creatividad se compone de tres elementos, tanto a nivel individual como organizacional, como se muestra en la figura 8.

Figura 8. Los tres componentes para la generación del pensamiento creativo

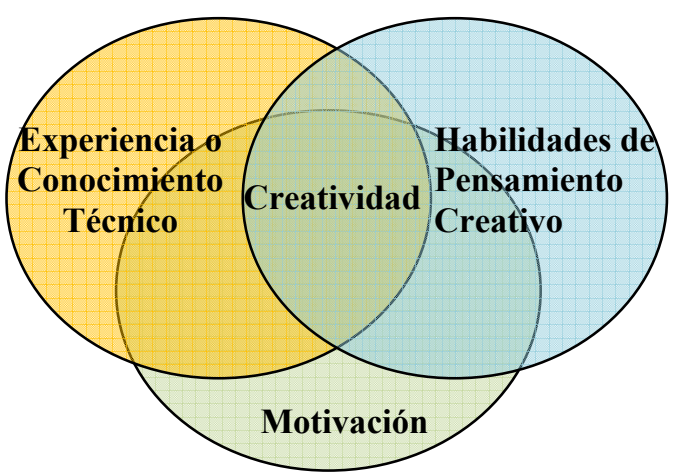

Fuente: La intersección de la Creatividad. Amabile 1988.

En la figura 8, se puede observar los tres elementos necesarios para la generación del pensamiento creativo. Son la experiencia en el tema, habilidades de pensamiento creativo y la motivación. En el punto central es donde se da la creatividad más abundante 0 alta del individuo o grupo pequeño de personas. Todo individuo, para generar creatividad, debe tener algo de estos tres componentes.

\section{O. López, M. Blanco \& S. Guerra}


El primer componente es el de la experiencia y refiere a los conocimientos técnicos que la persona tenga acerca del problema a resolver, que se considere un experto acerca del tema a analizar.

El segundo componente son las habilidades de pensamiento creativo y trata de lo capacitado que debe estar el individuo para generar creatividad, que tenga tolerancia a la ambigüedad, que sea arriesgado en su toma de decisiones y tolerancia a la frustración o fracaso, y lo desarrollado que se encuentre sobre técnicas de flexibilidad cognoscitiva e independencia intelectual.

El tercero es la motivación y ésta se divide es dos áreas: la motivación intrínseca (interna) y extrínseca (externa). La primera refiere a la pasión con la cual el individuo enfrenta el problema a resolver y del sentido de reto que representa para él este desafío, el deseo interno de satisfacción. El segundo es el reto que representa por tener un objetivo a lograr externo a su persona, como por ejemplo, si hubiese alguna remuneración para el caso ganador, o la presión de no perder el trabajo.

Este último componente, Amabile (1988) lo considera el más importante de los tres, ya que para generar un espacio de pensamiento creativo para la resolución de problemas, está directamente influenciado a la motivación del individuo para hacerlo. Aunque sea un experto en la materia o tenga desarrolladas sus habilidades creativas, si no hay una motivación interna o externa, difícilmente resultará exitoso.

\section{Resultados.}

De la base de datos antes mencionada de la Canieti, se realizó el análisis para las 34 empresas seleccionadas de la población meta. El cluster de tecnologías de la información en Nuevo León fue el elegido para este estudio. La encuesta es dirigida a los directores 0 gerentes generales de éstas empresas. Se aplica como base la Escala de Lickert y se utilizó la herramienta de correo electrónico para hacerla llegar a los actores de la investigación. En ciertos casos se realizó la entrevista presencial.

En base a lo anterior, se validó el modelo de gestión de la innovación propuesto en este artículo de investigación, además de medir el impacto de la creatividad organizacional en dicho modelo. Se dispuso a realizar un

\section{Creatividad Organizacional \& PyMEs}


análisis de fiabilidad (alfa de cronbach) para cada uno de los seis constructos. La cantidad de preguntas por variable se detallan en la tabla (en la figura 7 viene descrito lo que significa el código OM y el resto). Además se realizó un análisis de regresión lineal múltiple para obtener la prueba $F$, significancia, anovas, Durbin-Watson, colinealidad, Pearson, entre otros.

Tabla 3. Resultados del Análisis de Fiabilidad de la Muestra

\begin{tabular}{|c|c|c|c|c|c|c|c|c|c|c|}
\hline Variables & $\mathrm{N}$ & $\begin{array}{c}\text { Items } \\
\text { Iniciales }\end{array}$ & $\begin{array}{c}\text { Media } \\
\text { Global }\end{array}$ & Media & Varianza & $\begin{array}{c}\text { Desviación } \\
\text { Tipica }\end{array}$ & $\begin{array}{c}\text { Alfa } \\
\text { Cronbach }\end{array}$ & gl & $\begin{array}{c}\text { Chi-cuadrada } \\
\text { Friedman }\end{array}$ & $\begin{array}{c}\text { Signifi- } \\
\text { cancia }\end{array}$ \\
\hline OM & 34 & 9 & 3.741 & 33.676 & 61.498 & 7.842 & 0.889 & 33.000 & 4.318 & 0.000 \\
\hline CR & 34 & 9 & 3.349 & 30.147 & 45.766 & 6.765 & 0.857 & 33.000 & 6.852 & 0.000 \\
\hline I\&D & 34 & 9 & 3.356 & 30.205 & 73.380 & 8.566 & 0.883 & 33.000 & 3.589 & 0.001 \\
\hline DP & 34 & 6 & 3.250 & 19.500 & 24.439 & 4.943 & 0.790 & 33.000 & 17.017 & 0.000 \\
\hline EO & 34 & 10 & 3.147 & 31.470 & 74.196 & 8.613 & 0.884 & 33.000 & 2.937 & 0.002 \\
\hline EC & 34 & 9 & 3.366 & 30.294 & 64.759 & 8.047 & 0.900 & 33.000 & 10.085 & 0.000 \\
\hline MODELO & 34 & 53 & 3.341 & 177.117 & 1390.895 & 37.294 & 0.962 & 52.000 & 7.212 & 0.000 \\
\hline
\end{tabular}

Fuente: Elaboración Propia

Dentro de la tabla 3, se eliminaron varios ítems para aumentar el valor de alfa, volviéndose a correr el análisis tanto individualmente como modelo completo. Resultó el análisis con un alfa de $96 \%$. Se deduce, a partir de esto que el modelo es muy confiable para proceder a realizar el análisis de regresión lineal múltiple.

Se observa que la media global es de 3.34 lo que significa que existe todavía un gap entre el proceso de gestión de la innovación ideal vs lo que la industria de Tl esta haciendo para crear innovación en sus productos

Tabla 4. Resultados de la Regresión Lineal Múltiple

\begin{tabular}{|c|c|c|c|c|c|c|c|c|c|c|c|c|}
\hline $\begin{array}{l}\text { Modelo } \\
\text { Completo }\end{array}$ & N & Variables & $R$ & $R^{2}$ & $R^{2}$ corregida & gl & $\begin{array}{l}\text { Durbin- } \\
\text { Watson }\end{array}$ & & & & & \\
\hline Resumen & 34 & 6 & 0.913 & 0.833 & 0.828 & 1 & 1.979 & & & & & \\
\hline ANOVA & Media $^{2}$ & $\begin{array}{l}\text { "F" en la } \\
\text { Anova }\end{array}$ & \begin{tabular}{|c|}
$\begin{array}{c}\text { Significancia en } \\
\text { la Anova }\end{array}$ \\
\end{tabular} & & & & & & & & & \\
\hline Regresión & 313.097 & 164.255 & 0.000 & & & & & & & & & \\
\hline \multirow{2}{*}{$\begin{array}{l}\text { Análisis de } \\
\text { Pearson }\end{array}$} & \multicolumn{2}{|c|}{ Coeficientes no estandarizados } & \begin{tabular}{|c|}
$\begin{array}{c}\text { Coeficientes } \\
\text { estandarrizodos }\end{array}$ \\
\end{tabular} & \multirow[t]{2}{*}{$t$} & \multirow{2}{*}{ Sig. } & \multicolumn{2}{|c|}{$\begin{array}{c}\text { Intervalo de confianza } \\
\text { para B al } 95 \%\end{array}$} & \multicolumn{3}{|c|}{ Correlaciones } & \multicolumn{2}{|c|}{ Estadisticos de colinealidad } \\
\hline & $B$ & Error tip. & Beta & & & Limite inferior & Limite superior & Ordencero & Parcial & Semiparcial & Tolerancia & $\mathrm{FIV}$ \\
\hline 180 & 0.861 & 0.067 & 0.913 & 12.816 & 0.000 & 0.725 & 0.998 & 0.913 & 0.913 & 0.913 & 1.000 & 1.000 \\
\hline
\end{tabular}

Fuente: Elaboración Propia

\section{O. López, M. Blanco \& S. Guerra}


La tabla 4 indica que, según el análisis de Pearson la variable que justifica el modelo es la "lyD" ya que el $91 \%$ de la variabilidad del modelo es soportado por esta variable, como lo indica el valor de "R".

La regresión o correlación entre las variables independientes contra la dependiente se valida, como lo indica el Durbin-Watson con un valor de 1.97. La anova muestra buena independencia entre las variables independientes, porque solamente es una variable la que justifica el modelo con su valor de 164.25 .

No existe colinealidad ya que solamente una variable es la que justifica el modelo por su valor FIV de 1. Además la Beta es positiva con un alto valor y la Tstudent y su significancia, de igual manera, son relevantes para justificar aún más la importancia de la variable "lyD" en el modelo propuesto.

La tabla 5 muestra que, dentro del análisis de Pearson las variables hasta el momento excluidas son las otras cinco que conforman el modelo propuesto. La otra variable que obtuvo buen resultado es la OM, pues tiene buena significancia y correlación parcial. Aunque la EC y DP también muestra cierta colinealidad propositiva, pero se tendría que esperar ha contrastar con la lyD y alguna otra que obtenga buena variabilidad, y por último la CR y EO muestran un punto más alejadas de las otras tres variables restantes.

Tabla 5. Resultados de la Regresión Lineal Múltiple

\begin{tabular}{|c|c|c|c|c|c|c|c|}
\hline \multicolumn{8}{|c|}{ VARIABLES EXCLUIDAS } \\
\hline \multirow[b]{2}{*}{ Modelo } & \multirow[b]{2}{*}{ Beta dentro } & \multirow[b]{2}{*}{$\mathbf{t}$} & \multirow[b]{2}{*}{ Sig. } & \multirow{2}{*}{$\begin{array}{c}\text { Correlación } \\
\text { parcial }\end{array}$} & \multicolumn{3}{|c|}{ Estadísticos de colinealidad } \\
\hline & & & & & Tolerancia & FIV & $\begin{array}{c}\text { Tolerancia } \\
\text { mínima }\end{array}$ \\
\hline OM & -0.285 & -0.958 & 0.345 & -0.167 & 0.058 & 17.389 & 0.058 \\
\hline CR & 0.077 & 0.188 & 0.852 & 0.033 & 0.031 & 31.928 & 0.031 \\
\hline DP & 0.166 & 0.488 & 0.629 & 0.086 & 0.045 & 22.363 & 0.045 \\
\hline EO & 0.172 & 0.392 & 0.698 & 0.069 & 0.027 & 37.084 & 0.027 \\
\hline EC & -0.117 & -0.389 & 0.700 & -0.069 & 0.057 & 17.507 & 0.057 \\
\hline
\end{tabular}

Fuente: Elaboración Propia

\section{Discusión.}

En base a lo visto en los resultados, se concluye que:

1. El constructo de lyD es la que explica la variabilidad del modelo, por lo que el empresario PyME está consciente que esta área es la clave para innovar en sus productos.

\section{Creatividad Organizacional \& PyMEs}


2. La Creatividad Organizacional todavía no se ve como una herramienta crucial para generar innovación, aunque se considera algo importante. Se deduce que los empresarios no la consideran como un proceso operacional para las muestras de PyMEs en este estudio, y menos que deba de gestionarse orgánica y sistemáticamente.

3. La Orientación al Mercado fue la segunda mejor evaluada, por lo que se deduce que realmente sí toman en cuenta al cliente para innovar, pero no es su soporte para definir directrices en sus estrategias de mercado.

4. La Eficiencia Operacional es importante, pero no genera grandes expectativas al estar buscando nuevas tecnologías para desarrollar sus productos, ya que creen más en la habilidad de la gente para producir algo que en las nuevas tecnologías.

5. El Diseño del Producto no es parte importante para este sector, ya que obtuvo evaluación baja, se piensa que no es tomada en cuenta, pues no necesitan imagen para comercializar sus productos.

6. La Eficiencia Comercial no fue de las mejores evaluadas por lo que se deduce que las estrategias de comercialización no es el fuerte en esta industria, por lo que no utilizan métodos complicados ni rebuscados de venta.

El cluster de T.I. en NL esta muy familiarizado con los apoyos gubernamentales (InnovaPyME, InnovaTec, Prolnnova, entre otros) sobre proyectos específicos de innovación, por lo que lo sensibiliza mucho en la investigación y desarrollo. Además la percepción de estos empresarios, es que para innovar en productos innovadores necesitas lyD en tu organización. Cabe mencionar que esta industria, por naturaleza, está muy familiarizada con la innovación por su giro de mercado.

Algunos autores han comentado que la mayoría de los modelos estudiados (las generaciones de modelos de innovación) parecen estar orientados hacia empresas de gran tamaño, que disponen de departamentos internos de lyD, dejando a un lado las empresas de menor tamaño que operan a través de procesos más informales y que no cuentan con departamentos propios de ingeniería o de investigación y desarrollo.

En el Modelo propuesto se incluye un nuevo proceso denominado "Creatividad Organizacional" que busca la exploración y generación de ideas en la organización, tanto internamente como externa a ella, a partir de las

\section{O. López, M. Blanco \& S. Guerra}


necesidades del mercado. En esta etapa, como la llama Forrest (1991) preinnovación o de exploración, se generan ideas y se evalúan opciones, por lo que la creatividad y el recurso al conocimiento externo a la empresa resultan vitales.

Por consiguiente, la investigación y desarrollo todavía resulta como importante y vital por los empresarios para que se dé la innovación en el producto, pero se sugiere seguir apostándole a la creatividad organizacional, ya que ésta permite explotar el potencial creativo de los empleados dentro de las compañías que día a día trabajan con los productos obteniendo ideas creativas y que éstas, puedan resultar más efectivas para la creación de productos innovadores. La creatividad debe ser vista como un proceso sistemático, evaluando y desafiando la mejora de los productos que manufactura la empresa, evitando así el desperdicio que se suelen tener algunas compañías, al no explotar el potencial creativo de sus empleados.

\section{Recomendaciones.}

Se espera que en México se empiece a estudiar y profundizar en este tema tan relevante, ya que la economía actual del país demanda cada vez más empresarios y emprendedores que le inviertan a la Creatividad e Innovación, que vayan en busca de esos nichos de mercado que no han sido explorados hasta el momento.

Se busca que estas ideas de nuevos negocios no busquen competir por querer una porción del mercado que ya está repartido, si no que tengan en la mira los mercados que no han sido explotados hasta el momento. Además de saber que las PyMEs en México alcanzaron los cuatro millones (Conacyt, 2007) de empresas, el país debería estar construyendo un sistema de innovación nacional, basado en la economía del conocimiento, por lo que se debe comprometer con el fortalecimiento del capital humano, la tecnología, la defensa de la propiedad intelectual (patentes) y la innovación.

Por lo tanto, se recomienda para investigaciones futuras buscar si la variable Liderazgo es parte fundamental para que la creatividad e innovación se den más en las PyMEs. A su vez, seguir capacitando más a los empresarios para que vean a creatividad organizacional como un proceso orgánico y sistemático, que se debe gestionar para obtener mejores resultados. Además de quitar del pensamiento de la gente que este tema es algo abstracto que no se puede medir.

\section{Creatividad Organizacional \& PyMEs}




\section{Referencias.}

Amabile T., et al., 1996. Assessing the work environment for creativity. The Academy of Management Journal, Vol. 39, No 5, octubre, pp. 1154- 1184.

Amabile, T., 1995, 23 de Enero. Creativity Killers [Stevens, Tim]. Industry Week (US), sec. ISSN/ISBN 00390895, p. 63.

Amabile, T., 1998. A model of creativity and innovation in organizations. Research in Organizacional Behiavor. Vol.10 pp. 123-167.

Arraut, L., 2007. Elementos Clave para generar la capacidad emprendedora para el desarrollo de las organizaciones innovadoras. Recuperado el 02 de Noviembre del 2007, de http://ihm.ccadet.unam.mx/virtualeduca2007/pdf/179LAC.pdf......(producto del proyecto de investigación doctoral).

BCG, 2006. Innovation Survey Report. The Boston Consulting Group.

De Bono, E., 1994. El pensamiento creativo. México D.F: Paidos Plural-Titulo Original Serious Creativity. Using the Power of Lateral Thinking to Create New Ideas.

European Commission, 2004. Innovation Management and the Knowledge-Driven Economy. Bruselas: ECSC-EC-EAEC.

Forrest, J. E., 1991. Models of the Process of Technological Innovation. Technology Analysis \& Strategic Management, vol. 3, n 4, pp. 439-453.

Kim, W. C. \&. M., 2005. La estrategia del océano azul. Boston M.A US.: Harvard Business School Press.13.

Kim, W. C. \&. M., 2005, Feb. Navigating Toward Blue Oceans. Optimize, pp. 44-52.

Kline, S. y Rosenberg, N., 1986. An Overview of Innovation, in the Positive Sum Strategy: Harnessing Technology for Economic Growth. Washington, D.C., National Academy Press, pp. 275-305

Nahir, Y. \& Gómez, I., 2007. Estrategias para la generación de innovación en PyMEs. Recuperado el 22 de Febrero del 2008, de: http://fido.palermo.edu/servicios_dyc/encuentro2007/02_auspicios_publicaciones/actas_diseno/articul os_pdf/A5024.pdf

Rothwell, R., 1994. Towards the fifth-generation innovation process. International Marketing Review, vol. 11, $n^{\circ}$ 1. pp. 7-31.

Takeuchi, H. y Nonaka, I., 1986. The new product development game. Stop running the relay race and take up rugby. Harvard Business Review, enero-febrero, pp. 137-146.

Velasco, E., Zamanillo, I., \& Gurutze, M. (nd). Universidad de La Rioja. Recuperado el 10 de Marzo de 2008, de:

http://dialnet.unirioja.es/servlet/fichero_articulo?codigo=2499438\&orden $=0$.

\section{O. López, M. Blanco \& S. Guerra}

